\title{
Auf dem Weg zum nachhaltigen Frieden?
}

\author{
Aufgaben der Friedensforschung zu Beginn des 21. Jahrhunderts*
}

\author{
Volker Rittberger**
}

\begin{abstract}
This article advocates a process-based conception of sustainable peace and outlines an agenda for research that is committed to the concept of sustainable peace. It sketches major trends in the global incidence, prevention and management of violent conflict, and highlights several challenges for peace research at the beginning of the $21^{\text {st }}$ century, including religions in violent conflict and peace processes, (re-) construction of state capacities in failing or failed states, arms control and disarmament as well as climate change and violent conflict. The article presents preliminary findings and charts the territory for further research in these areas.
\end{abstract}

Keywords: Sustainable peace, religions, statehood, arms control and disarmament, climate change

Nachhaltiger Frieden, Religionen, Staatlichkeit, Rüstungskontrolle und Abrüstung, Klimawandel

\section{Förderung des postgradualen Masterstudien- gangs „Friedensforschung und Sicherheits- politik"}

$\mathrm{M}$

it ihrer Entscheidung vom Dezember 2001, die Einrichtung des postgradualen Masterstudiengangs „Friedensforschung und Sicherheitspolitik“ (Master of Peace and Security Studies - MPS) an der Universität Hamburg zu fördern, begab sich die Deutsche Stiftung Friedensforschung auf ein förderpolitisches Neuland für die Friedens- und Konfliktforschung. Mit der Trägerschaft durch einen Kooperationsverbund deutscher Friedensforschungsinstitute und der Zertifizierung der Studienabschlüsse durch die Universität Hamburg wies der Studiengang eine durchaus ungewöhnliche - wenn nicht gar gewagte - organisatorische Grundlage auf, die sich freilich trotz der Vielzahl der beteiligten Akteure über die Jahre hinweg bewährt zu haben scheint. Es ist insbesondere das bleibende Verdienst des damaligen Direktors des IFSH und meines Vorgängers im Amt des Stiftungsvorsitzenden, des leider allzu früh verstorbenen Prof. Dieter S. Lutz, dass diese in der Rückschau so gelungene Innovation auf den Weg gebracht werden konnte.

Im Rahmen ihres Programms zur Struktur- und Nachwuchsförderung stellte die Stiftung über einen Zeitraum von sechs Jahren Fördermittel in einer Gesamthöhe von 1,2 Millionen Euro für diesen Studiengang bereit. Das Kernstück der Fördermaßnahme bildeten 15 Studienstipendien, die für jedes Studienjahr zur Verfügung standen. (Die letzte Stipendientranche wurde allerdings über zwei Jahrgänge verteilt, um den Übergang in die finanzielle Eigenständigkeit zu erleichtern.) Darüber hinaus deckte die Stiftung mit ihrer Unterstützung einen Großteil der Organisations- und Betreuungskosten für das Studienangebot.

Von Anfang an wurde das interdisziplinär angelegte Studienprogramm, soweit ich dies als Außenstehender beobachten

\footnotetext{
* Festvortrag anlässlich der Verleihung der Masterdiplome an den 6. Jahrgang der Absolventinnen und Absolventen des Studiengangs Master of Peace an Security Studies (MPS) am 8. Oktober 2008 in der Universität Hamburg.

** Prof. Dr. Volker Rittberger ist Vorsitzender der Deutschen Stiftung Friedens forschung (DSF).
}

konnte, mit einem außerordentlichen Engagement seitens der Lehrenden und der Studiengangverantwortlichen umgesetzt. Es war für mich deshalb keine Überraschung, dass die von der DSF veranlasste Zwischenevaluierung dem Masterstudiengang im vergangenen Jahr ein hervorragendes Zeugnis ausstellte.

Die anhaltend große Nachfrage nach Studienplätzen verdeutlicht, dass sich der Studiengang national wie international ein hohes Ansehen erworben hat. Für die Stiftung ist es höchst erfreulich, feststellen zu können, dass die Initiativförderung zu einer nachhaltigen Strukturbildung der Friedens- und Konfliktforschung in Hamburg beigetragen hat. Ich bin deshalb auch optimistisch, dass es - nicht zuletzt dank der Aufgeschlossenheit und Unterstützung der Universität Hamburg - gelingen wird, den Masterstudiengang nach Auslaufen der Förderung durch die DSF auf eine dauerhafte und finanziell gesicherte Grundlage zu stellen. Bei dieser Gelegenheit darf ich auch daran erinnern, dass die Stiftung mit der Carl Friedrich von Weizsäcker-Stiftungsprofessur „Naturwissenschaft und Friedensforschung“ ein weiteres „Leuchtturmprojekt“ an der Universität Hamburg fördert, das mit dem Masterstudiengang „Friedensforschung und Sicherheitspolitik“ eng verbunden ist und dem die DSF als Initialstifter für die Zukunft ebenfalls ein großherziges Wohlwollen der Universität und des Landes Hamburg wünscht. Zusammen mit dem IFSH - und weiteren universitären Forschungseinheiten wie z.B. die Arbeitsgemeinschaft Kriegsursachenforschung (AKUF) oder die Forschungsstelle Kriege, Rüstung und Entwicklung (FKRE) - ist somit eine „kritische Masse“ an Friedensforschungseinrichtungen allein an der Universität Hamburg entstanden. Diese Bündelung wissenschaftlicher Kompetenz wird nicht zuletzt aufgrund ihrer disziplinären Vielseitigkeit und der darin angelegten interdisziplinären Kooperationsmöglichkeiten dazu beitragen können, wegweisende Konzeptionen und Strategien für eine nachhaltige Friedenssicherung zu entwickeln und die friedenspolitische Praxis wissenschaftlich zu begleiten. Damit habe ich mir das Stichwort gegeben, um zum Thema meines Vortrags „Auf dem Weg zum nachhaltigen Frieden?“ überzugehen und auf wichtige Aufgabenstellungen einzugehen, denen sich die Friedens- und Konfliktforschung zumal in Deutschland heute und in der überschaubaren Zukunft gegenüber sieht. 


\section{Nachhaltiger Frieden ? - Aufgaben für die Friedensforschung}

\subsection{Was bedeutet „nachhaltiger Frieden“?}

Der friedenswissenschaftliche Diskurs ist seit jeher von grundlegend verschiedenen Politikverständnissen geprägt und kennt mithin auch unterschiedliche Friedensbegriffe. Um zu erörtern, was unter dem Terminus „nachhaltiger Friede“ zu verstehen ist, beschränke ich mich darauf, den Blick auf eines dieser Politikverständnisse zu richten, das in der Wissenschaft weiteste Verbreitung gefunden hat: das prozessuale Politikverständnis - Politik definiert als die verbindliche Zuweisung von Werten in und zwischen Gesellschaften.

Der Ausgangspunkt des prozessualen Politikverständnisses ist die Annahme einer Omnipräsenz von Konflikten, die auch ein Definitionsmerkmal des Sozialen ist: „Gesellschaft ist Konflikt um menschliche Lebenschancen “. ${ }^{1}$ Das prozessuale Politikverständnis basiert auf einem weiten Begriff des Konflikts „verstanden als Positionsdifferenzen über Werte“. ${ }^{2}$

Für Ralf Dahrendorf ist das von einigen Klassikern der politischen Theorie als konfliktfrei portraitierte ,gute Gemeinwesen’ „das furchtbare Bild einer perfekten Gesellschaft“"3. Für ihn ist Gesellschaft nur geschichtlich zu begreifen, d.h. als zukunftsoffenes, nie abgeschlossenes Projekt. Ein dauerhaft gültiges Modell des ,guten Gemeinwesens' kann es daher nicht geben. Aller Fortschritt beruht „auf der Vielfalt und Widersprüchlichkeit der menschlichen Gesellschaft, d.h. darauf, im Widerstreit der Normen und Gruppen die jeweils annehmbare Lösung zu finden, um sie sogleich wieder kritisch zu relativieren". 4

Die Konzeptualisierung von Konflikten als Positionsdifferenzen über Werte beinhaltet „die Möglichkeit, den Akzent auf den Austragungsmodus [von Konflikten] zu rücken“. ${ }^{5}$ Geht man von der Omnipräsenz von Konflikten aus, so zeigt sich, dass nur ein kleiner Teil davon unter Anwendung oder Androhung von Gewalt ausgetragen wird. Ein großer, wenn nicht der größte Teil von menschlichen Lebenschancen wird hingegen gewaltlos zugeteilt.

Vertreter eines prozessualen Politikverständnisses sind sowohl der Überzeugung, dass jeder Konflikt gewaltfrei ausgetragen werden kann, als auch der Auffassung, dass ein gewaltfreier Konfliktaustrag in jedem Fall vorzugswürdig ist. Nach dieser Auffassung dominiert nicht ein Basiskonflikt (sei es zwischen Klassen oder aller um Macht) das soziale Leben. Vielmehr treten Konflikte angesichts ihrer Vielzahl und Vielfalt zumindest teilweise unabhängig voneinander auf, sie können mithin voneinander unabhängig betrachtet und stückwerkstechnisch - kompetitiv oder kooperativ - bearbeitet werden.

1 Dahrendorf, Ralf 1972: Konflikt und Freiheit: Auf dem Weg zur Dienstklassengesellschaft. München: Piper, S. 7.

2 Czempiel, Ernst-Otto 1981: Internationale Politik. Ein Konfliktmodell. Paderborn: Schöningh, S. 199.

3 Dahrendorf, Ralf 1974: Pfade aus Utopia. Arbeiten zur Theorie und Methode der Soziologie. 3. Auflage. München: Piper, S. 272-276, hier S. 275.

4 Ebenda, S. 276.

5 Czempiel, Ernst-Otto 1981: Internationale Politik. Ein Konfliktmodell. Paderborn: Schöningh, S. 201.
Da Konflikte zum Definitionsmerkmal von Gesellschaft gehören, kann Frieden nicht die Abwesenheit von Konflikten bedeuten. Das Auftreten von Konflikten in und zwischen Staaten und Gesellschaften ist damit gewissermaßen deren Lebenselixier und steht mit dem prozessual verstandenen Frieden nicht in Widerspruch. Nur der gewaltsame Austrag dieser Konflikte kennzeichnet die Abwesenheit von Frieden. Daher muss es das Ziel von Friedenspolitik sein, im Konfliktfall einem gewaltsamen Konfliktaustrag vorzubeugen oder dessen Wahrscheinlichkeit zu reduzieren. Dieter S. Lutz spitzte diese Anforderung an eine erfolgreiche Friedenspolitik folgendermaßen zu:

„Krieg und Frieden sind ebenso wenig natürliche Alternativen, wie Krieg nicht wirklich eine normale Fortsetzung der Politik mit anderen Mitteln ist. Vornehmste Aufgabe von Politik ist es vielmehr, Krieg zu verhüten, nicht ihn zu führen. Situationen, die als Alternative nur Krieg zulassen, darf es nicht geben. Treten sie ein, hat die Politik versagt". ${ }^{\text {}}$

Frieden im prozessualen Verständnis ist, mit Czempiels Worten, „ein Prozessmuster des internationalen Systems, das gekennzeichnet ist durch abnehmende Gewalt und zunehmende Verteilungsgerechtigkeit" “. Frieden ist somit immer auch ein gerechter Frieden verstanden als ein nie abgeschlossener Prozess, der in innerstaatlicher und zwischenstaatlicher Hinsicht nicht nur auf die Vermeidung von Gewaltanwendung, sondern auch - in den Worten der jüngsten EKD-Friedensdenkschrift - auf „die Förderung von Freiheit und kultureller Vielfalt sowie den Abbau von Not gerichtet ist" ${ }^{8}$ Frieden im prozessualen Verständnis umfasst damit nicht nur das bloße Überleben, sondern eine bestimmte Qualität menschlichen Lebens durch die Verringerung der Disparitäten in der Verteilung materieller und immaterieller Güter und des Zugangs zu ihnen.

Die vorherigen Ausführungen legen nahe, dass ein prozessualer Friedensbegriff auch der Vorstellung von einem „nachhaltigen Frieden“ am nächsten kommt: Frieden als Prozess soll zum Wohle der zukünftigen Generationen verstetigt werden, kann aber nie zu einem Ende kommen. Zusätzlich erweitert der nachhaltige Frieden den prozessualen Frieden um die sozial-ökologische Gerechtigkeitsdimension: der ökologisch tragfähige Umgang mit natürlichen Ressourcen und deren Bewahrung für zukünftige Generationen. Dieses bedeutet auch, dass an einem friedlichen Zusammenleben beständig gearbeitet werden muss, selbst wenn in einem Hier und Jetzt augenscheinlich Frieden ,erreicht' worden ist. Die Friedensforschung, so brachte es Dieter S. Lutz treffend auf den Punkt, müsse sich aus der Existenzerhaltung und -entfaltung des Menschen bestimmen, „Frieden hat auch insofern immer einen Nachhaltigkeitsanspruch. “9

6 Lutz, Dieter S. 2003: ,Der Friede als Ernstfall' oder ,das Recht des Stärkeren'. In: Lutz, Dieter S. (Hrsg.) Die Stärke des Rechts gegen das Recht des Stärkeren politische und rechtliche Einwände gegen eine Rückkehr des Faustrechts in die internationalen Beziehungen. Baden-Baden: Nomos, 17-34, hier S. 18

7 Czempiel, Ernst-Otto 1986: Friedensstrategien: Systemwandel durch internationale Organisationen, Demokratisierung und Wirtschaft. Paderborn Schöningh, S. 47.

8 Evangelische Kirche in Deutschland (EKD) 2007: Aus Gottes Frieden leben - für gerechten Frieden sorgen. Gütersloh: Gütersloher Verlagshaus, S. 54

9 Lutz, Dieter S. 2002: Friedensforschung und Nachhaltigkeitsforschung - Partnerinnen auf dem Weg in die Zukunft. S + F: Vierteljahresschrift für Sicherheit und Frieden 20 (4), S. 201-202, hier S. 201. 


\subsection{Kontinuität und Wandel von Unfrieden und Frieden}

Die Definition dessen, was unter einem nachhaltigen Frieden zu verstehen ist, erfolgt stets unter Bezugnahme auf die aktuelle geschichtliche Ausgangslage. Wichtige Orientierungspunkte hierfür bietet z. B. der 2005 erstmals erschienene Human Security Report..$^{10}$ Diese Studie kommt zu dem Ergebnis, dass sich die globale Lage im Bezug auf gewaltsame Konflikte deutlich verbessert hat. So ist die Zahl der bewaffneten Konflikte seit Anfang der 1990er Jahre um etwa 40 Prozent rückläufig. Die Zahl beigelegter gewaltsamer Konflikte (43) liegt in diesem Zeitraum deutlich über der neu ausgebrochener (28). Auch die Zahl der internationalen Krisen und der Verbrechen gegen die Menschlichkeit sind in diesem Zeitraum deutlich zurückgegangen. Das Volumen des internationalen Waffenhandels lag 2005 um ein Drittel niedriger als 1990. Schließlich hat sich auch die Zahl der Flüchtlinge um rund 45 Prozent verringert. Der Human Security Brief 2007 weist zudem daraufhin, dass seit 1998 entgegen der öffentlichen Wahrnehmung auch der transnationale Terrorismus rückläufig ist. ${ }^{11}$

Die Ursache dieser insgesamt positiven Entwicklung vermuten die Autoren des Berichts in der gewachsenen Zahl internationaler Friedensmissionen, insbesondere der Vereinten Nationen. Auch wenn ein gesicherter Kausalzusammenhang bislang nicht wissenschaftlich erwiesen ist, so spricht doch einiges dafür, dass die veränderten Rahmenbedingungen im internationalen System nach dem Ende des Kalten Krieges dazu geführt haben, dass die Bereitschaft in der Staatengemeinschaft gewachsen ist, von den VN geführte oder mandatierte Interventionen mit dem Ziel der Beendigung von Gewaltkonflikten und des postconflict peacebuilding zu unterstützen.

Ein diese Entwicklung begünstigender Faktor kann in der Emergenz einer als heterarchisch zu bezeichnenden Weltordnung gefunden werden. ${ }^{12}$ Der Terminus „Heterarchie“ verweist auf ein „drittes“ Weltordnungsprinzip zwischen Anarchie und Hierarchie. Es handelt sich um eine Alternative zu anarchischen Selbsthilfesystemen in einer Welt von Großmächten und Kleinstaaten auf der einen Seite und formal oder de facto hierarchischen Systemen, also Weltstaat bzw. hegemoniale oder gar imperiale Steuerung, auf der anderen. ${ }^{13}$ Mit „Heterarchie“ wird die Existenz eines dichter werdenden Netzes von inklusiven, multipartistischen Institutionen und Praktiken des Weltregierens beschrieben, die von öffentlichen und privaten Akteuren zur kollektiven, regelgeleiteten Bearbeitung von transsouve-

10 Vgl. Human Security Report 2005. War and Peace in the 21st Century. New York et al.: Oxford University Press.

11 Vgl. Human Security Brief 2007: Dying to Lose. Explaining the Decline in Global Terrorism. Burnaby: Simon Fraser University.

12 Vgl. Rittberger, Volker 2008: Global Governance: From ,Exclusive‘ Executive Multilateralism to Inclusive, Multipartite Institutions. Tübinger Arbeitspapiere zur internationalen Politik und Friedensforschung Nr. 52. Tübingen: Institut für Politikwissenschaft, Universität Tübingen; Rittberger, Volker/ Huckel, Carmen/ Rieth, Lothar/Zimmer, Melanie 2008: Inclusive Global Institutions for a Global Political Economy, in: Rittberger, Volker/ Nettesheim, Martin (Hrsg.): Authority in the Global Political Economy. Basingstoke: Palgrave Macmillan, S. 11-54.

13 Vgl. Holsti, Kalevi J. 1992: Governance without Government: Polyarchy in Nineteenth-Century European International Politics, in: Rosenau, James N./ Czempiel, Ernst Otto (Hrsg.): Governance without Government: Order and Change in World Politics. Cambridge: Cambridge University Press, 30-57, hier S. 56f. ränen Problemen durch horizontale Politikkoordination und -kooperation geschaffen und aufrechterhalten werden.

Durch das Entstehen inklusiver, multipartistischer Institutionen des Weltregierens wie z.B. des Global Fund to Fight HIV/ AIDS, Malaria and Tuberculosis oder des Global Compact - um nur zwei der zahlreichen globalen öffentlich-privaten Partnerschaften oder Multistakeholder-Initiativen im Bereich der Global Governance herauszugreifen - wird deutlich, dass der lange Zeit vorherrschende Exekutivmultilateralismus von Staaten, ganz zu schweigen vom Unilateralismus sich zunehmend selbst übernehmender Großmächte offenbar nicht die letzte Antwort in Bezug auf die institutionelle Lösung kollektiv zu bearbeitender Probleme auf globaler Ebene ist.

Gerade die Einbeziehung privater Akteure, von denen Gewaltkonflikte häufig ausgehen, und die damit für sie einhergehende Möglichkeit zur Teilhabe an Steuerungsprozessen, kann einer direkten Verringerung des weltweiten Gewaltpotenzials zuträglich sein. Es kann davon ausgegangen werden, dass die größere Vernetzung öffentlicher und privater Akteure in einer heterarchischen Ordnung gewaltsamen Konfliktaustrag zwischen eben diesen Akteuren für diese kostenintensiver und mithin unwahrscheinlicher macht. Die indirekte Wirkung der Heterarchie entfaltet sich in einer effektiveren und legitimeren Behandlung transsouveräner Probleme und damit einer zuverlässigeren Verregelung von Konflikten. Transsouveräne Probleme mit hohem Konfliktpotenzial, wie z. B. weltweite Pandemien und der globale Klimawandel, lassen sich schließlich nur erfolgreich und nachhaltig bearbeiten, wenn alle „Problemproduzenten“, also auch nichtstaatliche Akteure, gemeinsam daran beteiligt werden. ${ }^{14}$

Leben wir angesichts dieser Beobachtungen heute also in einer friedlicheren Welt?

Die Autoren des Human Security Report äußern sich keineswegs enthusiastisch über ihre zuvor genannten Befunde: Von Entwarnung könne keine Rede sein, da man es noch immer mit einer Vielzahl akuter Gewaltkonflikte weltweit zu tun habe. Vielmehr habe sich der Charakter dieser Konflikte geändert, die heute vergleichsweise geringere Opferzahlen aufwiesen und auch mit anderen Mitteln und Waffen ausgetragen würden. Die Frage des sich wandelnden Charakters der Gewaltkonflikte ist Gegenstand einer seit mehreren Jahren anhaltenden internationalen Debatte, auch in der deutschen Friedensforschung, über die „neuen Kriege“, auf die ich freilich hier nicht näher eingehen kann ${ }^{15}$. Es sei hier nur beispielhaft auf die neueste Forschung von Sven Chojnacki verwiesen, der in seiner Analyse des Wandels der Gewaltformen im internationalen System von 1946-2006 einige der zentralen Thesen über die „neuen Kriege“ als empirisch und methodisch problematisch identifiziert ${ }^{16}$.

14 Vgl. Müller, Harald 2008: Wie kann eine neue Weltordnung aussehen? Wege in eine nachhaltige Politik. Frankfurt/M.: Fischer.

15 Vgl. z. B. Herfried Münkler 2002: Die neuen Kriege, Reinbek: Rowohlt und Mary Kaldor 1999: New and Old Wars: Organized Violence in a Global Era, Cambridge: Polity Press

16 So spricht etwa Münkler, sicherlich der prominenteste Vertreter der These der „neuen Kriege“ in Deutschland, von sogenannten ,alten' zwischenstaatlichen Kriegen als „Auslaufmodell“, während Chojnacki in seiner Forschung verdeutlicht, dass „militärische Konfrontationen, Sicherheitsdilemmata und Rüstungsspiralen zwischen Staaten nach wie vor Probleme regionaler und internationaler Politik sind (v.a. im Nahen und Mittleren Osten sowie in Südasien)“ (Chojnacki 2008: 33). 
Ich möchte an dieser Stelle nun einige Themenfelder herausgreifen, die mit Blick auf das Konzept des nachhaltigen Friedens eine besondere Aufmerksamkeit der Friedens- und Konfliktforschung verdienen.

\subsection{Aufgaben der Friedensforschung zu Beginn des 21. Jahrhunderts}

\section{A) Die Rolle von Religionen in Gewaltkonflikten und Friedenspro- zessen}

Eines der Themengebiete, denen die DSF eine Forschungsinitiative gewidmet hat, ist die Rolle von Religionen in Gewaltkonflikten und Friedensprozessen. Die Friedensforschung steht nicht erst mit der tagespolitischen Dominanz von Akten zumindest scheinbar religiös motivierten Terrors und der damit verbundenen Bedrohungswahrnehmungen vor der Frage, welche Bedeutung Religionen in der Entstehung, aber auch in der Prävention oder Beilegung von Gewaltkonflikten zukommt.

Noch Mitte der 1990er Jahre formulierte Samuel Huntington seine bekannte These vom "Zusammenprall der Kulturen“,17 der die nach dem Zweiten Weltkrieg dominierende, hoch militarisierte Form des politisch-ökonomischen Systemkonflikts ablösen würde. Heute wird diese eindimensionale Kausalität zwischen Kultur oder Religion und Gewaltkonflikten von der Forschung weitgehend abgelehnt. ${ }^{18}$ Die Empirie zeigt, dass religiöse Differenzen in höchst seltenen Fällen Ursachen von Gewaltkonflikten sind, sondern allenfalls verschärfende Wirkung auf ursprünglich politisch oder wirtschaftlich motivierte Konfrontationen entfalten ${ }^{19}$. In der Analyse von Bürgerkriegen lässt z.B. die schiere Zahl von Bürgerkriegen zwischen Angehörigen ein und derselben Religion Glaubensunterschiede als Ursache für Bürgerkriege in quantitativen Untersuchungen bedeutungslos werden. ${ }^{20}$ Das Kriegsrisiko einer Gesellschaft wird offenkundig nicht wesentlich von ihrer religiösen Binnenstruktur beeinflusst. ${ }^{21}$

Im Hinblick auf eine nachhaltige Friedenssicherung sind die weiteren Befunde dieser Untersuchungen von Bedeutung, dass religiöse Differenzen von Seiten politischer Eliten instrumentalisiert werden können. Hasenclever und De Juan sprechen von einem Rückgriff auf religiöse Traditionen als Strategie, um Gefolgschaft zu mobilisieren und Gewalt gesellschaftlich zu legitimieren. ${ }^{22}$ Religiöse Unterschiede und religiöse Unzufriedenheit wirken mithin nicht als Brandursache, sondern als Brandbeschleuniger. Für die Friedensforschung stellt sich demnach die Frage, wie sich religiöse Traditionen vor politischer Instrumentalisierung schützen (lassen). Analysen religiöser Friedensbewegungen deuten darauf hin, dass sich instrumentalisierungsresistente Glaubensgemeinschaften vor allem durch

17 Huntington, Samuel P. 1998: The Clash of Civilizations and the Remaking of World Order. New York: Simon \& Schuster.

18 Vgl. z.B. Said, Edward W. 2001: The Clash of Ignorance, in: The Nation 273(12), S. 11-14.

19 Vgl. Barnes, L. Philip 2005: Was the Northern Ireland Conflict Religious?, in: Journal of Contemporary Religion 20(1), S. 55-69; Hasenclever, Andreas/Alexander De Juan 2007: Religionen in Konflikten. Eine Herausforderung für die Friedenspolitik, in: Aus Politik und Zeitgeschichte 6/2007, S. 10-16.

20 Vgl. Fox, Jonathan 2004: Religion, Civilization, and Civil War. 1945 Through the New Millennium. Lanham, MD u.a.: Lexington Books

21 Vgl. Hasenclever/ De Juan, a.a.O. (Anm. 19).

22 Vgl. ebenda. vier Merkmale auszeichnen: religiöse Aufklärung im Sinne des Respekts vor der Komplexität religiöser Überlieferungen ${ }^{23}$, strukturelle Toleranz in Form von institutionalisierten moderaten Diskursen ${ }^{24}$, ein hohes Autonomiepotenzial gegenüber Staat und Gesellschaft ${ }^{25}$ sowie eine diversifizierte innerreligiöse Öffentlichkeit. ${ }^{26}$

Nicht weniger interessant ist die Frage, unter welchen Bedingungen Religionen entgegen gängiger Annahmen auch de-eskalierenden Einfluss auf die Dynamik von Gewaltkonflikten haben können. ${ }^{27}$ Hier wird das politisch wirksame Friedenspotenzial religionsbasierter Akteure zum Forschungsthema, wie es schon aus dem religiös motivierten, gewaltlosen Wirken einzelner wie Mahatma Gandhi, Martin Luther King oder Desmond Tutu bekannt ist. Dass religiöse Überzeugungen und Werte als verbindendes Element zwischen Konfliktparteien auch Lösungswege ebnen können, zeigen in neuerer Zeit etwa die Mitwirkung der Evangelischen Kirche in der friedlichen Revolution der DDR, die ruandischen Muslime, die sich 1994 als einzige Gruppe dem Genozid enthielten, oder die von der Weltkonferenz der Religionen für Frieden (WCRP) ins Leben gerufenen „Religious Councils“, die in Bosnien-Herzegowina, im Kosovo, in Liberia und Sierra Leone eine vermittelnde Funktion übernahmen. ${ }^{28}$

Diese Untersuchungen zeigen, dass der vielfach unterstellte kausale Nexus zwischen Religionen und Gewaltkonflikten einer Neubewertung bedarf, und dass die Friedensforschung gerade im Hinblick auf die Untersuchung des Zusammenhangs zwischen Religionen und Frieden künftig weitere Lücken unseres Wissens schließen muss.

\section{B) Wieder- und Neuaufbau staatlicher Strukturen in Krisengebieten}

Gewaltkonflikte in Form von internen oder Bürgerkriegen, der heute vorherrschenden Art von Gewaltkonflikten, brechen vor allem dort aus, wo Staatlichkeit von vielen Bruchstellen bedroht ist oder staatliche Strukturen durch einen Zerfallsprozess gekennzeichnet sind - in so genannten failing states oder

23 „Arbeiten zum Verhältnis von Religion und Gewalt zeigen regelmäßig, dass Glaubensüberschreitungen vor allem dann eskalierend wirken, wenn sie selektiv und mit radikalem Absolutheitsanspruch interpretiert werden" (ebenda, S. 14).

24 „Im Kontext andauernder politischer oder sozialer Konflikte können oftmals radikale religiöse Botschaften institutionalisiert werden und damit die Instrumentalisierungsanfälligkeit religiöser Gemeinschaften erhöhen. In Nigeria beispielsweise wurden Eliten in ihren Verteilungskämpfen um die Gewinne der nigerianischen Ölwirtschaft jahrzehntelang von muslimischen und christlichen Geistlichen mit radikalen Interpretationen unterstützt“ (ebenda, S. 15).

25 „Etablierte Religionsgemeinschaften mit einer großen Zahl von Gläubigen brauchen Ressourcen: Gotteshäuser, Geistliche und karitative Leistungen müssen finanziert werden; die Ausübung und Vermittlung des Glaubens erfordert entsprechende institutionelle und gesetzliche Rahmenbedingungen. Hierzu gehört beispielsweise die Möglichkeit, religiöse Feste öffentlich zu feiern oder Religionsunterricht anzubieten. Sind religiöse Gemeinschaften beim Zugriff auf diese Ressourcen von der Willkür und dem Wohlwollen staatlicher oder gesellschaftlicher Akteure abhängig, besteht die Gefahr, dass sich ihre Führer zur Legitimierung staatlicher Gewaltpolitik einspannen lassen, um das institutionelle Überleben ihrer Gemeinschaften zu sichern“ (ebenda, S. 15).

26 Vgl. ebenda.

27 Vgl. Weingardt, Markus A. 2008: Religion als Friedensressource. Potenziale und Hindernisse, in: Wissenschaft \& Frieden 26(3): S. 26-29; Göller, Kerstin 2008: Jihad, Waffenstillstand oder Frieden mit Israel? Der Zusammenhang von politischer Gewalt und religiöser Konflikt-Interpretation im Nahostkonflikt, in: Religion - Konflikt - Frieden: Ausgewählte Beiträge zur Jahrestagung 2007 des Forschungsverbundes Religion und Konflikt vom 16. bis 18. November in Bonn. Frankfurt am Main: Gemeinschaftswerk der Evangelischen Publizistik.

28 Vgl. Weingardt, a.a.O. (Anm. 27). 
failed states. ${ }^{29}$ Diese Konflikte haben zudem häufig grenzüberschreitende Auswirkungen und können ganze Regionen destabilisieren.

Die Beilegung dieser Art von Gewaltkonflikten und der sich daran anschließende Aufbau einer stabilen Friedensordnung, im Englischen als post-conflict peacebuilding bezeichnet, sind wichtige Themenstellungen, denen die Friedensforschung künftig eine verstärkte Aufmerksamkeit widmen sollte. Hierbei geht es vor allem um angepasste Strategien der Friedenskonsolidierung, für die sehr unterschiedliche politische, kulturelle, wirtschaftliche und soziale Faktoren in die Analyse einbezogen werden müssen. So stellt sich beispielsweise die Frage der Abfolge (sequencing) und Koordinierung von Maßnahmen wie (Wieder-) Aufbau von Institutionen der Verwaltung und Rechtspflege, Herstellung von öffentlicher und privater Sicherheit (security sector reform), wirtschaftlicher Wiederaufbau, Partizipation und Demokratisierung bis hin zu Dialogstrukturen zwischen den Konfliktparteien und langfristig angelegten Versöhnungsprozessen. Auch hat sich in der Forschung die Erkenntnis durchgesetzt, dass die Durchführung von freien Wahlen am Anfang eines Prozesses der Friedenskonsolidierung kontraproduktiv sein kann, weil sie sich u.U. wieder konfliktverschärfend auswirken. Der (Wieder-)Aufbau von Staatlichkeit wird häufig überlastet, indem westliche Legitimationsmuster zur unbedingten Grundlage erhoben werden. ${ }^{30}$

Andreas Mehler kritisiert eben diesen „state bias“, also die Sichtweise auf den Staat als einzigem „Heilsbringer“ im Prozess der Friedenskonsolidierung. ${ }^{31}$ Er weist darauf hin, dass im direkten Gegensatz zum europäischen Verständnis staatliche Sicherheitsorgane in vielen Staaten Afrikas oft dadurch auffällig werden, dass sie eher „Unsicherheitsproduzenten“ sind. Gleichzeitig gelingt es nichtstaatlichen Akteuren nicht selten, mindestens für einen Teil der Bevölkerung Schutz anzubieten. Mehler weist darauf hin, dass sich die Friedensforschung in diesem Zusammenhang von vermeintlich klaren Dichotomien wie „staatlich - nichtstaatlich“ (häufig gleichgesetzt mit „gutböse“) verabschieden sollte. Anstelle der erzwungenen Einführung und Aufrechterhaltung von Gewaltmonopolen könnten Friedenseinsätze stärkeres Augenmerk auf die Optimierung von legitimen Gewaltoligopolen legen. ${ }^{32}$

Die bisherigen Erfahrungen mit friedenskonsolidierenden Aufbaumaßnahmen können zudem, gemessen an den hochgesteckten Zielsetzungen, nicht durchweg positiv bewertet werden. Erfolgreichen Friedenskonsolidierungsprozessen wie in Namibia, Mozambique oder Guatemala stehen weniger zielführende Missionen in Ländern wie Haiti und Ost-Timor gegenüber, in denen Rückfälle in Destabilisierung und Gewalt zu beobachten waren, sowie auch Missionen, die den Erwartungen an eine erfolgreiche Stabilisierung nicht gerecht werden konnten - Afghanistan und Somalia. ${ }^{33} \mathrm{Zu}$ berücksichtigen sind ferner

29 Vgl. Schneckener, Ulrich (Hrsg.) 2004: States at Risk. Fragile Staaten als Sicherheits- und Entwicklungsproblem. Berlin: Stiftung Wissenschaft und Politik.

30 Vgl. Schetter, Conrad/Katja Mielke 2008: Staatlichkeit und Intervention in Afghanistan, in: Die Friedens-Warte 83(1), S. 71-96, hier S. 90.

31 Mehler, Andreas 2008: Mehr Analyse, mehr Mut zu Kooperation: Peacekeeper, staatliche und nichtstaatliche Gewaltakteure in Afrika, in: Die Friedens-Warte 83 (1), S. 45-70, hier S. 45.

32 Ebenda, S. 64.

33 Rittberger, Volker 2008b: Frieden durch Staatlichkeit? - Einführung, in: Die Friedens-Warte 83(1), S. 11-24, hier S. 16f. so genannte Sekundärkonflikte, die vor allem dann auftreten, wenn sich die Wahrnehmung der Interventionskräfte durch die lokale Bevölkerung ändert und die anfangs wohlwollende Stimmung durch konkrete Erfahrungen und Feindbilder ins Gegenteil umschlägt.

Die internationale Politik steht somit vor einer zweifachen Herausforderung: Es gilt zum einen, dem weiteren Zerfall von fragilen Staaten durch koordinierte Maßnahmen vorzubeugen, zum anderen den Wieder- oder Neuaufbau staatlicher Strukturen in Nachkriegsgesellschaften in angepassterer Weise zu fördern. ${ }^{34}$ Dabei wird in der neueren Forschung darauf hingewiesen, dass der Erfolg friedenskonsolidierender Maßnahmen mit einer dauerhaften und soliden Einbindung lokaler Akteure (local ownership) erheblich steigt. ${ }^{35}$ Das Beispiel des internationalen Engagements in Afghanistan verdeutlicht, dass internationale Missionen in Post-Konflikt-Ländern dazu neigen, Parallelstrukturen zu errichten, die staatliche Aufgaben erfüllen, ohne dass zu deren schrittweiser Übernahme befähigte lokale Kapazitäten aufgebaut werden. ${ }^{36}$ Genau diese Entwicklung verhindert, dass lokale Beamte, Richter und Polizeikräfte den Staat mit Inhalt und Eigenverantwortung ausfüllen - und damit zur Schaffung stabiler staatlicher Strukturen und letztlich nachhaltigen Friedens beitragen. Ebenso trägt eine aktive Politik der Stärkung verantwortlicher Regierungsführung (good governance), z.B. durch den Aufbau funktionierender Rechtsordnungen in PostKonflikt-Staaten, erheblich zum Erfolg friedenskonsolidierender Maßnahmen bei.

\section{C) Die Notwendigkeit einer aktiven Abrüstungspolitik und präven- tiven Rüstungskontrolle}

Es mag Sie vielleicht überraschen, dass ich zu den zentralen Herausforderungen auch ein klassisches Themenfeld der Friedens- und Konfliktforschung zähle, das allerdings in den letzten Jahren nicht mehr die ihr gebührende Aufmerksamkeit seitens der Regierungen und Parlamente, aber auch der Öffentlichkeit erhalten hat: die Rüstungskontroll- und Abrüstungspolitik. In der jüngeren Vergangenheit waren es vor allem die Besorgnisse über die Atomprogramme des Iran und Nordkoreas, die der Öffentlichkeit die Gefahren der Verbreitung von Massenvernichtungswaffen vor allem an nichtdemokratisch regierte Staaten (und an nichtstaatliche Gewaltakteure) erneut vor Augen geführt haben. Nur wenig öffentliche Beachtung fand hingegen die geradezu beispiellose Aufrüstungsdynamik der letzten Jahre, die von einer Lähmung, wenn nicht gar einer Aushöhlung der multilateralen Rüstungskontroll- und Abrüstungspolitik begleitet wird. Nach neuesten Berechnungen des Stockholm International Peace Research Institute (SIPRI) erreichten die Militärausgaben im Jahr 2007 die nahezu unvorstellbare Höhe von rund 1,34 Billionen US-Dollar; im Vergleich zu 1998, also vor zehn Jahren, bedeutet dies einen Anstieg um 45 Prozent ${ }^{37}$. Die heutigen Rüstungsausgaben überschreiten somit selbst die Höchststände aus den Zeiten des Kalten Krieges.

\footnotetext{
34 Vgl. ebenda.

35 Vgl. Schetter/Mielke, a.a.O. (Anm. 30).

36 Ebenda, S. 90.

37 SIPRI Yearbook 2008: Armaments, Disarmament and International Security, S. 10. $45 \%$ der Gesamtausgaben im Jahr 2007 entfielen auf die Vereinigten
} Staaten von Amerika. 
Die multilaterale Rüstungskontroll- und Abrüstungspolitik, die in Zeiten der gerade auch von Deutschland unterstützten Politik der Entspannung des Ost-West-Konflikts ihre Bewährungsproben durchaus erfolgreich bestanden und bis Mitte der 1990er Jahre einen vielversprechenden Aufschwung genommen hatte, erlebt spätestens seit den Terroranschlägen vom 11. September 2001 einen steten Niedergang. Die westlichen Staaten müssen sich zu Recht die Frage gefallen lassen, was sie selbst zur Förderung der Nichtverbreitung von Massenvernichtungswaffen, aber auch von konventionellen Waffen und vor allem ihrer Abrüstung beigetragen haben. Die Weigerung der USA und anderer Kernwaffenstaaten, den Vertrag über das vollständige Verbot von Kernwaffenversuchen in Kraft treten zu lassen, und die Entwicklung immer kleinerer Atomsprengköpfe erhöhen nicht gerade die Aussichten auf abrüstungspolitische Durchbrüche. Die bestehenden Atomwaffenarsenale werden von den Kernwaffenstaaten also nicht nur instand gehalten, sondern technologisch modernisiert, und ihre militärischstrategische Einsatzfähigkeit wird zumindest teilweise gezielt vorbereitet. Einen beträchtlichen Teil der Verantwortung für diese Entwicklung trägt zweifellos die Bush-Administration, die sich vom - wenngleich immer schon tendenziell instrumentellen - Multilateralismus der amerikanischen Außen- und Sicherheitspolitik weitgehend lossagte und auf eine Politik des Unilateralismus und der unangefochtenen militärischen Stärke setzte $^{38}$.

Auf jeden Fall drängt sich für die Friedensforschung die Frage auf, wie zukünftig mit einer weitaus größeren Zahl von Nuklearmächten und ggf. auch zum Einsatz von Massenvernichtungswaffen fähigen nichtstaatlichen Akteuren umzugehen sein wird und ob internationale Regelwerke das Risiko eines Einsatzes oder eines Missbrauchs dieser Waffen verhindern können. Einen Ansatzpunkt bietet die ursprünglich von den USA ausgehende und von der G8 aufgenommene Initiative der Cooperative Threat Reduction, deren Effektivität aber Zweifeln unterliegt. Es bleibt die Einsicht, dass die internationalen Rüstungskontrollregime dringend einer strukturellen Anpassung und kreativen Weiterentwicklung bedürfen.

Des Weiteren scheint es mir von zunehmender Bedeutung zu sein, die Dynamik neuer Technologieentwicklungen im Hinblick auf deren potenzielle militärische Nutzbarkeit in Augenschein zu nehmen. Zu nennen wären in erster Linie die Biotechnologie und die Nanotechnologie. Verschiedene Studien haben in den vergangenen Jahren auf das Risikopotenzial der Entwicklung neuartiger biologischer und (nano-)technologischer Waffen hingewiesen. ${ }^{39}$

38 Dies zeigt sich unter anderem in der sogenannten Bush-Doktrin, die Präemptivschläge rechtfertigt und die nukleare Option wieder jenseits der bloßen Abschreckung offen als Mittel der Kriegführung darstellt: „The new doctrine's approach grants regional nuclear-strike planning an increasingly expeditionary aura that threatens to make nuclear weapons just another tool in the toolbox. The result is nuclear pre-emption, which the new doctrine enshrines into official U.S, joint nuclear doctrine for the first time, where the objective no longer is deterrence through threatened retaliation but battlefield destruction of targets" (Kristensen 2005)

39 Vgl. Nixdorff, Kathryn et al. 2003: Biotechnology and the Biological Weapons Convention. Münster: agenda; Altmann, Jürgen 2006: Military Nanotechnology - Potential Applications and Preventive Arms Control. Abingdon/New York: Routledge; Altmann, Jürgen 2005: Nanotechnology and Preventive Arms Control, Forschung DSF Nr. 3, Osnabrück: Deutsche Stiftung Friedensforschung.
Allein an diesen Beispielen aus der Forschung wird deutlich, dass die Zeit reif ist, gestützt auf diese Art von Rüstungstechnologie-Folgenabschätzung die Initiative für eine aktivere und präventive Rüstungskontrollpolitik zu ergreifen ${ }^{40}$ oder die Präventionsbestimmungen bestehender Rüstungskontrollabkommen zu stärken bzw. auszubauen. ${ }^{41}$ Das Laserblendwaffenprotokoll von 1996 ist ein gutes Beispiel präventiver Rüstungskontrolle, da es mit dem Verbot der Entwicklung und Erprobung von Laserwaffen zum dauerhaften Blenden von Personen eine neue, militärisch nutzbare, aber bisher noch nicht eingeführte Technik verbietet.

Um Bedingungen für nachhaltigen Frieden zu schaffen, muss das Ziel darin bestehen, Rüstungskontrolle und Abrüstung im Aufgabenspektrum sowohl regionaler als auch globaler Institutionen mit sicherheitspolitischer Regierensverantwortung fester zu verankern. Bestehende Abrüstungsverpflichtungen, wie z.B. der Art. VI NVV müssen ernst genommen werden, um wieder Glaubwürdigkeit und Vertrauen herzustellen. Aus deutscher Sicht könnte ein wichtiges Signal in einer mit den USA (nach gehöriger Konsultation mit den NATO-Partnern) zu erreichenden Übereinkunft bestehen, die letzten noch auf deutschem Boden verbliebenen Atomwaffen abzuziehen.

\section{D) Klimawandel und Gewaltkonflikte}

Eine weitere Forschungsinitiative der DSF gilt den möglichen friedensgefährdenden Auswirkungen des anthropogenen Klimawandels. Damit verbunden ist die Aufforderung an die Friedensforschung, den Blick in die Zukunft zu richten sowie Modelle und Methoden zu entwickeln, um die durch den Klimawandel induzierten Risiken auszuloten und Wege einer vorbeugenden und zivilen Konfliktbearbeitung aufzuzeigen. Nach dem heutigen Stand der Erkenntnisse und den daraus abgeleiteten Szenarien, z.B. im jüngsten Gutachten des Wissenschaftliche Beirats der Bundesregierung Globale Umweltveränderungen (WBGU), wird der Klimawandel die Anpassungsfähigkeit vieler Gesellschaften überfordern. Man muss die These von drohenden Klimakriegen nicht teilen; gleichwohl stellen Wissenschaftler jedoch vermehrt systematische Zusammenhänge zwischen Klimawandel und gewaltträchtigem Konflikt her. Marc Levy von der Columbia University in New York zeigt in einer rückblickenden Untersuchung auf, dass sich das Risiko der Eskalation eines schwelenden Konflikts in einen Bürgerkrieg verdoppelt, wenn in der jeweiligen Region der Regen ausbleibt. ${ }^{42}$ Allerdings spricht einiges dafür, dass es sich hierbei eher um indirekte als direkte Kriegsursachen, also um eine Verschärfung bereits bestehender gewaltträchtiger Konfliktkonstellationen handelt. Politische, soziale oder ökonomische Konfliktfaktoren stehen ebenso in einer Wechselwirkung zum Klimawandel wie die Möglichkeiten zur Anpassung an die sich wandelnden Umweltbedingungen. Denn nicht in allen Fällen von Hungersnöten oder Dürreperioden kommt es zum Ausbruch eines Gewaltkonflikts. Der Klimawandel kann die vorhandenen Verteilungskonflikte um knappe Ressourcen wie

\footnotetext{
40 Vgl. Altmann, Jürgen 2008: Präventive Rüstungskontrolle, in: Die FriedensWarte 83(2-3), S. 105-126.

41 Vgl: ebenda, S. 110.

42 Vgl. Giles, Jim. (2007). Rainfall Records Could Warn of War, in: New Scientist
} 194 (2606), S. 12. 
Wasser oder geeignetes Acker- oder Weideland freilich noch potenzieren. Allgemein generalisierbare Zusammenhänge konnten allerdings bisher nicht hergestellt werden. Außerdem wird noch zu häufig nicht zwischen dem langfristigen Klimawandel und den Folgen direkt vom Menschen zerstörter Umwelt durch Übernutzung, wie z.B. durch Abholzung, Überfischung oder Überweidung oder Versalzung der Böden, unterschieden.

Der Wissenschaftliche Beirat der Bundesregierung Globale Umweltveränderungen identifiziert vier Konfliktkonstellationen, in denen als Folge des Klimawandels kritische Entwicklungen zu erwarten sind: (1) klimabedingte Degradation von Süßwasserressourcen; (2) klimabedingter Rückgang der Nahrungsmittelproduktion; (3) klimabedingte Zunahme von Sturm- und Wasserkatastrophen und (4) umweltbedingte Migration. ${ }^{43}$ Der Wissenschaftliche Beirat vermutet, dass diese Konfliktkonstellationen in verschiedenen Regionen der Welt in ähnlicher Ausprägung auftreten können, wie z.B. in der Sahelzone ${ }^{44}$ und in Zentralasien ${ }^{45}$. Betroffen sind vor allem Entwicklungsländer, da sie die Auswirkungen des Klimawandels weniger abfedern können und dort häufig ökonomische, soziale oder politische Konflikte bereits schwelen.

VN-Generalsekretär Ban-Ki Moon vermutet sogar, dass der erste gewaltsame Konflikt aufgrund des gegenwärtigen Klimawandels bereits lange läuft und schon Hunderttausende Tote gefordert hat - er meint damit den Konflikt in Darfur ${ }^{46}$. Dürren über 20 Jahre hinweg haben viele Nomaden mit ihren Herden vom Norden in den Süden gedrängt, wo sie in Konflikt mit den Ackerbauern um Land gerieten.

Obwohl gerade die These in Bezug auf Darfur als erstem „KlimaKrieg “ umstritten ist, da andere Faktoren wie ethnische Zugehörigkeit oder Land-/Bodennutzungen als bedeutsamer eingeschätzt werden, zeigen diese Entwicklungen und Befunde doch deutlich den bestehenden Forschungsbedarf auf. ${ }^{47}$ Gerade die Friedensforschung muss in diesem ,jungen' Bereich ihren Beitrag leisten, den Zusammenhängen zwischen Klimawandel und Gewaltkonflikten systematisch nachzugehen und, darauf gestützt, für eine nachhaltige, v.a. gewaltpräventive und die natürliche Umwelt der Menschen schonende Politik sinnvolle Empfehlungen zu erarbeiten.

Das Attribut „nachhaltig“ vor dem Terminus „Frieden“ gewinnt durch den Klimawandel und seine Folgen besondere Bedeutung. Kann man den Klimawandel als eine die Gewaltträchtigkeit von Konflikten potenziell verstärkende Ursache nicht

43 WBGU 2007: Welt im Wandel. Sicherheitsrisiko Klimawandel. Zusammenfassung für Entscheidungsträger, unter: http://www.wbgu.de/wbgu_jg2007_ kurz.pdf, 29.09.2008, S. 1.

44 „Klimawandel verursacht zusätzlichen Umweltstress und Gesellschaftskrisen (z. B. Dürren, Ernteausfälle, Trinkwasserknappheit) in einer schon heute durch schwache Staaten (z. B. Somalia, Tschad), Bürgerkriege (z. B. Sudan, Niger) und große Flüchtlingsströme (Sudan: über 690.000 Menschen; Somalia: über 390.000 Menschen) charakterisierten Region“ (ebenda, S. 3).

45 „Überdurchschnittliche Erwärmung und Gletscherschwund verschärfen Wasser-, Landwirtschafts- und Verteilungsprobleme in einer Region, die bereits durch politische und soziale Spannungen, Erstarkung islamistischer Bewegungen, Bürgerkrieg (Tadschikistan) und Auseinandersetzungen um den Zugang zu Wasser- und Energieressourcen charakterisiert ist" (ebenda, S. 4).

46 "Amid the diverse social and political causes, the Darfur conflict began as an ecological crisis, arising at least in part from climate change" (Ban, Ki Moon 2007: A Climate Culprit in Darfur, in: The Washington Post, 16. Juni 2007, A15.).

47 Vgl. Faris, Stephan 2007: The Real Roots of Darfur, in: The Atlantic Monthly 301 (4), S. 67. ausschließen, so müssen zwei Strategien in Betracht gezogen werden: Zum einen natürlich den Klimawandel selbst auf nationaler wie internationaler Ebene bekämpfen, insbesondere durch die Reduzierung der $\mathrm{CO}^{2}$-Emissionen; zum anderen die vom Klimawandel am stärksten beeinträchtigten Staaten in ihrer Anpassungsfähigkeit unterstützen. So fordern Fachleute, die Klimawandelproblematik in Peacebuilding-Konzepten und -Praktiken zu berücksichtigen bzw. einen angemessenen Umgang damit in sie einzubetten. ${ }^{48}$ Wichtig sind hierbei vor allem die Sensibilisierung der aufzubauenden Institutionen und der Öffentlichkeit in den betroffenen Staaten, die Vermittlung von notwendigem Fachwissen oder die bessere Vernetzung der verschiedenen regionalen und internationalen Maßnahmen.

Im Rückblick auf die hier kurz vorgestellten vier Problemfelder ist zu bedenken, dass diese nur einen Teil der Herausforderungen widerspiegeln, denen sich die zeitgenössische Friedensforschung mit ihren praxeologischen Ansprüchen gegenübersieht, vor allem wenn es ihr Ziel ist, nachhaltigen Frieden - verstanden als einen auch und gerade die Erwartungen nachwachsender Generationen an Gewaltvermeidung und Gewährleistung von sozialen und ökologischen Lebenschancen ernst nehmenden Prozess - zu fördern. Dennoch würde ihre vertiefte Erforschung ebenso wie die anderer, hier nicht genannter Problemfelder des Zusammenlebens in der Welt von heute der Friedensforschung weitere wichtige Erkenntnisse liefern, die als Grundlage für die Aufklärung interessierter Öffentlichkeiten und für Empfehlungen und Handlungsanleitungen an die politische Praxis dienen können.

Der Weg zum nachhaltigen Frieden, um auf den Titel dieses Vortrags zurückzukommen, ist dementsprechend eng mit ebenso nachhaltigen Anstrengungen in Wissenschaft und Politik verbunden.

\section{Schluss}

Es ist mir wichtig, zum Schluss meiner Ausführungen noch einmal darauf hinzuweisen, dass die Beispiele für wichtige Themengebiete der Friedens- und Konfliktforschung ohne Weiteres ergänzt werden können. Als Stichworte seien an dieser Stelle nur die Entwicklungen im Völkerrecht, insbesondere die Diskussion über den Wandel des Souveränitätsverständnisses und die Schutzpflicht der Staaten (responsibility to protect), die Anforderungen an die Neu- und Weiterentwicklung internationaler Governance-Strukturen - nicht zuletzt auch vor dem Hintergrund der aktuellen Finanzmarktkrise -, die Diskussion über „menschliche Sicherheit“ (human security) als neues Sicherheitsverständnis sowie nicht zuletzt die Weiterentwicklung einer zukunftsfähigen europäischen bzw. transatlantischen Friedens- und Sicherheitspolitik genannt. Die Friedensund Konfliktforschung steht bei begrenzten Kapazitäten - und dies gilt bedauerlicherweise auch für die Fördermöglichkeiten der DSF - vor der großen Aufgabe, sowohl im Kleinen bei der Bearbeitung konkreter gewaltträchtiger Konfliktkonstellationen als auch im Großen bei der Entwicklung nachhaltiger Friedensstrategien Antworten auf die Herausforderungen zu

48 Vgl. Smith, Dan/Vivekanda, Janani 2007: A Climate of Conflict - The Links between Climate Change, Peace and War. London: International Alert, S. 4. 
entwickeln, die sich zu Beginn des 21. Jahrhunderts stellen. Das Ziel, nachhaltige Friedensprozesse zu befördern, macht es notwendig, auch die Erwartungen nachwachsender Generationen an Gewaltvermeidung und der Erhaltung, besser noch: Stärkung von sozialen und ökologischen Lebenschancen mit einzubeziehen. Die Friedens- und Konfliktforschung steht hierbei unter dem Zugzwang, ihre Befunde auch für die friedenspolitische Praxis und für interessierte Öffentlichkeiten nutzbar zu machen, indem sie politische Handlungsoptionen und -szenarien aufzeigt. Auch der Masterstudiengang „Friedensforschung und Sicherheitspolitik“ liegt genau an dieser Schnittstelle zwischen Friedensforschung und politischer Praxis und bietet deshalb hervorragende Voraussetzungen für die Ausbildung wissenschaftlicher Fachkräfte, die für die Umsetzung nachhaltiger Friedensstrategien benötigt werden.

\title{
The Responsibilty to Protect - Norm im Entstehen?
}

\author{
Tim J. Aristid Müller-Wolf/ Patricia Schneider*
}

\begin{abstract}
In the 2005 World Summit Outcome Document, the Member States of the UN agreed to protect their populations from genocide, war crimes, ethnic cleansing and crimes against humanity and that the international community, through the UN, has the responsibility to use peaceful means yet is prepared to take coercive action, through the Security Council, if states fail to fulfil their responsibilities. However, some questions remain unanswered. Fear of misusage, the concept of sovereignty and the veto right are major obstacles for the implementation of the "Responsibility to Protect". Although the concept has gained support in the academic community, civil society, some governments and the UN it remains at present an "emerging norm" at most, rather than being the guiding principle of international relations or part of international law.
\end{abstract}

Keywords: Schutzverantwortung, Menschenrechtsschutz, Vereinte Nationen, UN-Sicherheitsrat

Responsibility to Protect, human rights protection, United Nations, UN Security Council

\section{Einführung}

Die Ereignisse nach dem Ende des Kalten Krieges zwangen die Staatengemeinschaft vor dem Hintergrund massiver Verletzungen von Menschenrechten und humanitärem Völkerrecht gerade in innerstaatlichen Konflikten, sich mit den Konzepten Souveränität und Humanitäre Intervention auseinanderzusetzen. Nach Möglichkeit sollte ein Konsens über Kriterien für die Rechtmäßigkeit einer Intervention gefunden werden. Diese Notwendigkeit wurde besonders durch die Berichte über Srebrenica und Ruanda, die im Auftrag der UN-Generalversammlung respektive des damaligen Generalsekretärs verfasst wurden und den mangelnden Schutz von Zivilisten in Konflikten beklagten, deutlich. Kofi Annan verlangte daher im Vorfeld des UN-Millenniumgipfels eine Antwort auf die Frage, wie mit dem Prinzip der Souveränität angesichts massiver Menschenrechtsrechtsverletzungen umzugehen sei. Diese wurde durch den damaligen kanadischen Premierminister Jean Chrétien auf eben diesem Gipfel angekündigt: Es sollte eine International Commission on Intervention and State Sovereignty (ICISS) eingerichtet werden, um einen tragfähigen Konsens zu erarbeiten. ${ }^{1}$ Die Kommission unter Vorsitz des ehemaligen australischen Außenministers Gareth Evans, der 1994 für den Aufsatz "Cooperative Security and Intrastate Conflict" in der

\footnotetext{
* Tim J. Aristid Müller-Wolf, Jurist und Master of Peace and Security Studies (MPS); Dr. phil. Patricia Schneider, wissenschaftliche Referentin am Institut für Friedensforschung und Sicherheitspolitik an der Universität Hamburg.

1 ICISS, The responsibility to protect: http://www.iciss.ca/pdf/CommissionReport.pdf, Mandat S. 81 .
}

Herbstausgabe der Zeitschrift Foreign Policy den Grawemeyer Prize for Ideas Improving World Order gewann und des algerischen Diplomaten Mohamed Sahnoun, UN-Sonderberater und UN-Sondergesandter, verfasste sodann ihren Bericht „The Responsibility to Protect“ (R2P oder RtoP, „Schutzverantwortung").

Der Bericht beinhaltete zunächst eine sprachliche Neujustierung, weg von einem Recht oder gar einer Pflicht zur humanitären Intervention hin zu einer Ausrichtung auf den Schutz von Menschen. Die Spaltung der UN-Generalversammlung in Staaten, die Interventionen im Namen der Menschenrechte befürworten und Staaten, die auf absoluter Souveränität bestehen, sollte so überwunden werden. Das Konzept sollte nicht im Gegensatz zum traditionellen staatenzentrierten Sicherheitsverständnis stehen, sondern vielmehr eine Antwort auf dessen Schwächen geben. Zwei Punkte ließen Staaten allerdings Zurückhaltung gegenüber diesem Konzept üben: Mögliche Auswirkungen auf die Souveränität und die Unglaubwürdigkeit der guten Absichten. Die Schutzverantwortung wurde gar als Euphemismus für angelsächsisches Hegemoniestreben gesehen. Die Aufnahme des Konzepts der Schutzverantwortung, das kurz nach 9/11 präsentiert wurde, in den UN wird unterschiedlich beurteilt. Die EU wurde kritisiert, das Konzept lediglich verbal zu unterstützen, anstatt es in konkrete Politikmaßnahmen umsetzen zu wollen. ${ }^{2}$

2 Gareth Evans verlangt stärkere Anstrengungen, www.crisisgroup.org/home/ index.cfm?id=4936\&l=1, Abruf am 15. 12. 2008 . 